\title{
Enhanced Refractometer Based on Periodically Tapered Small Core Singlemode Fiber
}

\author{
Pengfei Wang \\ Technological University Dublin, pengfei.wang@tudublin.ie \\ Gilberto Brambilla \\ University of Soughampton \\ Ming Ding \\ University of Southampton
}

See next page for additional authors

Follow this and additional works at: https://arrow.tudublin.ie/engscheceart

Part of the Electromagnetics and Photonics Commons

\section{Recommended Citation}

Wang, P., Brambilla, G., Ding, M., Lee, T., Bo, L., Semenova, Y., Wu, Q, Farrell, G.: Enhanced Refractometer Based on Periodically Tapered Small Core Singlemode Fiber. IEEE Sensors Journal, 2012, In Press. doi:10.1109/JSEN.2012.2216865

This Article is brought to you for free and open access by the School of Electrical and Electronic Engineering at ARROW@TU Dublin. It has been accepted for inclusion in Articles by an authorized administrator of ARROW@TU Dublin. For more information, please contact arrow.admin@tudublin.ie, aisling.coyne@tudublin.ie, gerard.connolly@tudublin.ie.

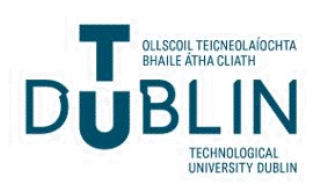




\section{Authors}

Pengfei Wang, Gilberto Brambilla, Ming Ding, Timothy Lee, Lin Bo, Yuliya Semenova, Qiang Wu, and Gerald Farrell 


\title{
Enhanced Refractometer Based on Periodically Tapered Small Core Singlemode Fiber
}

\author{
Pengfei Wang, Gilberto Brambilla, Ming Ding, Timothy Lee, Lin Bo, \\ Yuliya Semenova, Qiang Wu, and Gerald Farrell
}

\begin{abstract}
An all-fiber refractive index (RI) sensor with a simple configuration of periodical tapers is proposed and investigated experimentally. The proposed fiber RI sensor consists of a small core fiber sandwiched between two standard singlemode fibers, with tapers periodically fabricated along the small core fiber using a focused $\mathrm{CO}_{2}$ laser beam. Such a structure can be used for RI sensing by measuring the dip wavelength shift of the multimode interference within the small core fiber cladding. An average sensitivity of $226.6 \mathrm{~nm} / \mathrm{RIU}$ (RI Unit) has been experimentally achieved in the RI range from 1.33 to 1.38 . The refractometer is sensitive to temperature and an experimental investigation of this sensitivity is presented. It is found that the peak shift response has a linear variation with temperature; therefore, temperature dependence can be mitigated by a suitable RI correction process. The proposed RI sensor benefits from simplicity and low cost and achieves a competitive sensitivity compared with other existing fiber-optic sensors.
\end{abstract}

Index Terms-Fiber gratings, fiber optics, optical fiber device, optical fiber sensors.

\section{INTRODUCTION}

$\mathbf{O}$ PTICAL fiber refractive index (RI) sensors offer advantages such as immunity to electromagnetic interference, high sensitivity, fast response, small size and ease of fabrication. To date several types of optical fiber refractometers have been proposed for applications in growth areas for RI sensing [1]-[12]. The most common approaches are refractometers based on on a fiber Bragg grating [1]-[3], long period grating [4]-[6], surface plasmon [7]-[9], tapered microfiber [10]-[13],

Manuscript received May 23, 2012; revised August 11, 2012; accepted August 27, 2012. This work was supported in part by the National Access Program, Tyndall National Institute, Cork, Ireland. The work of P. Wang was supported by the Irish Research Council, co-funded by the MarieCurie Actions under FP7. The work of G. Brambilla was supported by the Royal Society (London) for his research fellowship. The work of $\mathrm{Q}$. Wu was supported by the Science Foundation Ireland under Grant 07/SK/I1200, Grant 11/TIDA/B2051, Grant 07/SK/I1200-STTF11, and Grant 07/SK/I1200ISTTF11. The associate editor coordinating the review of this paper and approving it for publication was Prof. Michael Schöning.

P. Wang is with the Optoelectronics Research Centre, University of Southampton, Southampton SO17 1BJ, U.K., and also with the Photonic Research Centre, Dublin Institute of Technology, Dublin 8, Ireland (e-mail: pw3y09@orc.soton.ac.uk).

G. Brambilla, M. Ding, and T. Lee are with the Optoelectronics Research Centre, University of Southampton, Southampton SO17 1BJ, U.K. (e-mail: gb2@orc.soton.ac.uk; md20d09@orc.soton.ac.uk; t1305@orc.soton.ac.uk).

L. Bo, Y. Semenova, Q. Wu, and G. Farrell are with the Photonic Research Centre, Dublin Institute of Technology, Dublin 8, Ireland (e-mail: bo.lin@mydit.ie; yuliya.semenova@dit.ie; qiang.wu@dit.ie; gerald.farrell@ dit.ie).

Color versions of one or more of the figures in this paper are available online at http://ieeexplore.ieee.org.

Digital Object Identifier 10.1109/JSEN.2012.2216865

bent fiber [14] and a singlemode fiber (SMF)-multimode fiber (MMF)-singlemode fiber [15]. As an alternative to these existing fiber refractometers, an SMF28 -small core -SMF28 (SSCS) fiber structure based fiber refractometer [16] has been proposed recently featuring a high sensitivity of $102 \mathrm{~nm} / \mathrm{RIU}$ at an RI $=1.324$. With respect to the other techniques, an SSCS provides a reliable high sensitivity sensor at low cost.

For RI sensing, the SSCS structure proposed in Ref. [16] needs strong multimode interference between the fiber cladding modes, thus the diameter of the cladding of the small core fiber in an SSCS structure should be small in order to achieve high sensitivity. Experimentally, an effective approach is to employ a small core fiber with a reduced cladding diameter achieved by etching with hydrofluoric acid, but this increases the fabrication difficulty and the induced surface roughness may lead to a significant discrepancy between experimental results and theoretical design [14]. Alternatively, fiber tapering can replace the chemical etching process; since light guided in a tapered fiber has a significant fraction of power propagating as an evanescent wave, the effective index of the guided mode is affected by the surrounding medium RI. It is well known that the fraction of power in the form of evanescent field, and thus its sensitivity to environmental changes, increases for smaller taper diameters.

Since Davis et al. [17] reported the first example of long period fibre grating (LPFG) written by $\mathrm{CO}_{2}$ laser irradiation in a conventional glass fiber in 1998, this method has been used to manufacture a variety of devices and sensors. Compared with the LPFG fabricated by a UV-laser exposure technique, the $\mathrm{CO}_{2}$ laser irradiation technique is much more flexible and low cost because no photosensitivity or sensitization such as hydrogen loading are needed. Moreover the $\mathrm{CO}_{2}$ laser irradiation process can be controlled to generate complicated grating profiles via the well-known point-to-point technique without the use of any expensive masks. Therefore this technique can be used to write LPFGs in almost all types of fibers including pure-silica photonic crystal fibers. In this paper, we report the experimental demonstration of a novel high sensitivity refractometric sensor based on multimodal interference in a singlemode-periodically tapered small core fiber-singlemode fiber structure (SPTS) capable of improving the sensitivity using a series of microtapers [18]. The small core fiber used in the experiments was a commercial fiber (Nufern S405 HP) with a core diameter of circa $2.5 \mu \mathrm{m}$ and a cut-off wavelength of $365 \mathrm{~nm}$. The schematic configuration of the SSCS used as the starting point to fabricate the SPTS used in the experiments 


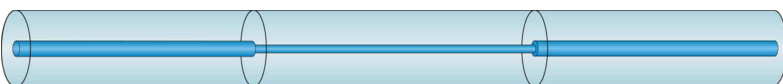

Fig. 1. Schematic of the SMF28-small core fiber-SMF28 fiber structure.

is shown in Fig. 1. Light propagation in such an SSCS fiber section has been simulated and analysed using an eigenmode analytical method presented in Ref. [16]. During the experimental verification, it was found that the power of the core mode guided in such an SSCS structure is low $(\sim-25 \mathrm{~dB})$ and can thus be ignored in the theoretical model, this supports the fact that only the multimode interference between the cladding modes is essential for the device to work and should be considered.

Ref. [18] has shown that if the multimode section is tapered in a conventional singlemode-multimode-singlemode (SMS) structure, then strong mode interference occurs within the tapered MMF section due to the focusing effects of the input section to the taper. Within the tapered MMF section, the excited modes of LP0m in the MMF core will be partly coupled to the high-order cladding modes at the beginning of the fiber taper region and this increases the fraction of power in the evanescent field within the region of MMF cladding. This phenomenon offers a possibility to increase the intensity of multimode interference of the cladding modes in an SSCS using a tapered structure. Furthermore, by using several concatenated tapers, a periodic taper structure is created as shown in Fig. 2, consisting of an input SMF, a periodically tapered small core fiber section and an output SMF. Such an SPTS structure offers the potential to achieve a higher sensitivity than a single taper alone due to the increased cladding mode interference induced by the multiple focusing effects of the input section of each of the tapers.

\section{Theoretical Analysis}

In our recent published work, a comprehensive theoretical analysis for an SSCS fiber structure has been presented using an eigenmode analytical method. The SSCS fiber with surrounding RI liquid can be treated as a three layer structure without a fundamental core mode guided in the core of the small core fiber. The good agreement between the calculated and measured results has shown that the theoretical eigenmode analytical method employed is an effective way to predict the performance of an SSCS fiber refractometer. Since in practice there is no fundamental core mode guided in the small core fiber, therefore the three layer fiber structure (core-cladding-surrounding RI) can be simplified as a two layer fiber structure, namely a cladding-surrounding RI structure. This offers a possibility to simulate the light propagation in the SSCS fiber structure using a wide angle-beam propagation method (WA-BPM) with cylindrical coordinates using a Padé $(3,3)$ approximate operator and perfectly matched layer (PML) boundary conditions which has been presented in the

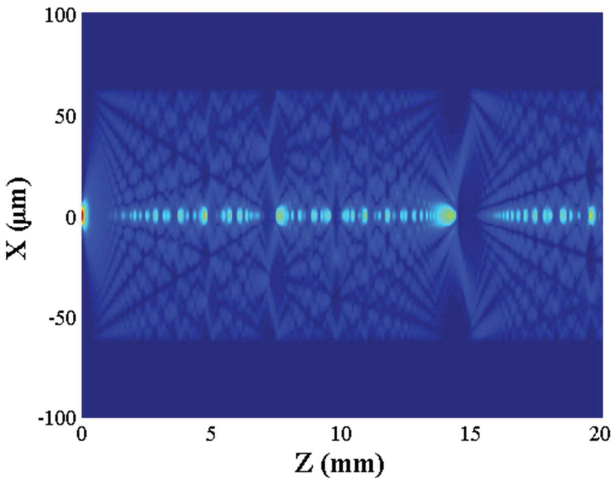

(a)

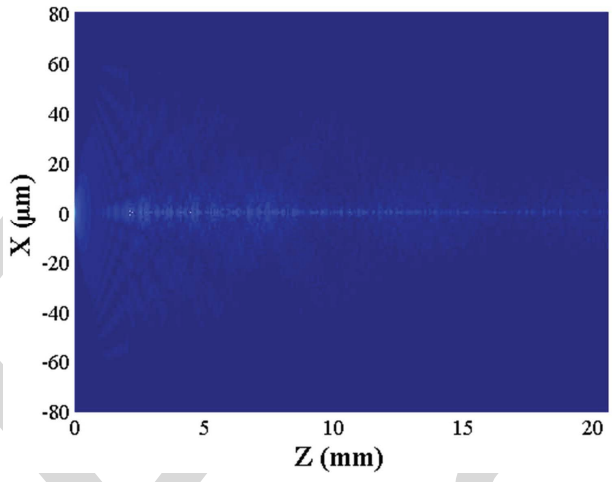

(b)

Fig. 3. Calculated amplitude of optical fields at a wavelength of $1550 \mathrm{~nm}$ using a WA-BPM for (a) SSCS fiber sandwiched between two standard SMFs, and (b) SPTS fiber with 28 microtapers with a waist diameter of $90 \mu \mathrm{m}$, sandwiched between two standard SMFs.

Ref. [18]. To prove the foundation of this approach, an alcoholbased graphite adhesive with high absorption coefficient was applied to the $2 \mathrm{~cm}$ long surface of the small core fiber sandwiched between two SMFs. Light transmitted in the SSCS fiber structure experienced a $\sim 25 \mathrm{~dB}$ attenuation, hence verifying that almost all of the light is actually transmitted in the cladding of the small core fiber.

The calculated amplitudes of the optical fields of both SSCS and SPTS fiber structures are presented in Fig. 3(a) and (b) respectively. Fig. 3(a) shows for reference the expected optical field at a wavelength of $1550 \mathrm{~nm}$ for an SSCS section, while Fig. 3(b) shows that weakly confinement occurs within the SPTS section due to the reduced size of fiber core diameter within the microtapers: at long wavelengths, beyond the cutoff wavelength, such as $1550 \mathrm{~nm}$, the core mode expands out into the surrounding silica cladding as the core diameter reduces. This results in a more efficient coupling with the fiber cladding modes compared with the SSCS fiber structure, which explains the non-adiabatic and highly lossy behavior of the SPTS fiber structure. Compared with the SSCS structure shown in Fig. 3(a), the optical power propagating through the fiber core of the SPTS structure (fig. 3(b)) is very low because of the enhanced multimode interference and weak confinement of the fiber core mode.

The peak wavelength shifts of both SSCS and SPTS fiber structures as a function of surrounding RI are calculated using a WA-BPM. The simulated results in figure 4 confirm that 


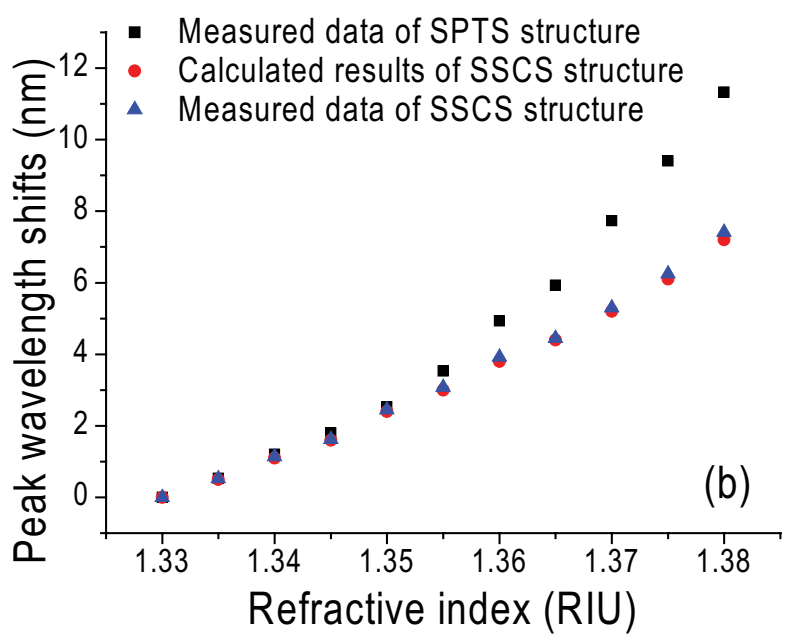

Fig. 4. Calculated peak wavelength shifts of the SSCS and SPTS fiber structures as a function of surrounding RI.

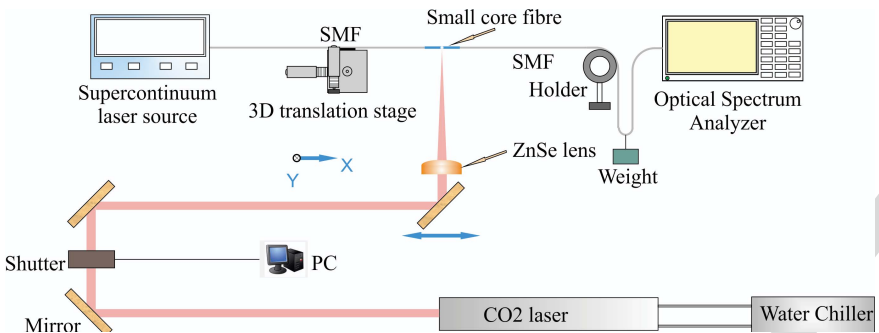

Fig. 5. Schematic of the fabrication and experimental setup.

as the surrounding RI increases the central wavelengths of both SSCS and SPTS fiber structures increase monotonically in an exponential fashion. The peak wavelength shift of the SPTS fiber structure is much higher than that of the SSCS fiber structure, confirming that the refractometer with a SPTS structure has a higher sensitivity than that with a SSCS fiber structure.

\section{FABRicAtion OF THE SPTS Fiber StRUCtURE}

The SSCS fiber sample used as the starting point for the SPTS was fabricated from a $21 \mathrm{~mm}$ length of Nufern S405 HP step index SMF which was stripped, cleaved and then spliced between two standard SMF28 fibers. As shown in the fabrication setup in Fig. 5, a $\mathrm{CO}_{2}$ laser (SYNRAD, Model: $48-2 \mathrm{KWL}$, with a maximum power 30 Watts at a wavelength of $10.6 \mu \mathrm{m}$ ) was employed to fabricate the tapered fiber. A $\mathrm{ZnSe}$ cylindrical lens with a focal length of $254 \pm 0.5 \%$ $\mathrm{mm}$ focused the $\mathrm{CO}_{2}$ laser beam to $\sim 200 \mu \mathrm{m}$. Beam movement was achieved using gold-coated mirrors on a motorized translation stage. A Labview program controlled the shutter opening and therefore the laser exposure time. One 3D translation stage was used to adjust the heating position within the small core fiber section, while a weight $(\sim 3.7 \mathrm{~g})$ was used to apply a constant tension to the SMF end of the SSCS structure. The small core fiber was exposed to the $\mathrm{CO}_{2}$ laser beam with an output power of $10.5 \mathrm{~W}$ for $120 \mathrm{~s}$ and tapering occurred because of the simultaneous localized heating of the fiber and tension applied to the end

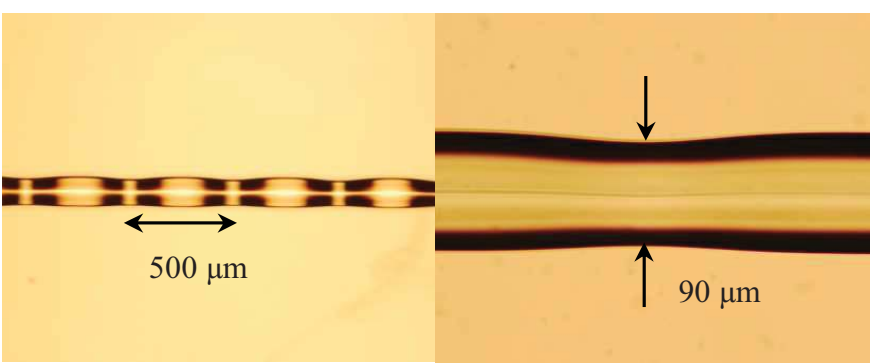

Fig. 6. Images of the small core fiber with periodic microtapers. The image was obtained with a microscope (Nikon Eclipse LV100) with a $5 \times$ (top) and $20 \times$ (bottom) objective, respectively.

of the SMF. Then the laser beam was translated a distance of $400 \mu \mathrm{m}$ along the fiber axis to irradiate and taper another segment of the fiber. This tapering process was repeated up to 20 times. Consequently, periodic tapers were created on the small core fiber, as shown in Fig. 6(a). Although the translation distance of the laser beam is $400 \mu \mathrm{m}$ along the fiber axis, the actual period of the tapers is circa $500 \mu \mathrm{m}$ due to the elongation of the fiber induced by tapering. The tensioning weight used during fiber processing was removed for subsequent refractive index measurements, with the fiber sample mounted instead on a "U" shaped stage. The detail of one of the resulting tapers is shown in Fig. 6(b), the waist diameter is circa $90 \mu \mathrm{m}$. The transmission spectra of the SPTS fiber structure were monitored and recorded during fabrication using a Supercontinuum source (Fianium Ltd, U.K.), which delivered $50 \mathrm{~nJ}$ light pulses over a broad range of wavelengths (450-1800 nm), and a high resolution (20 pm) optical spectrum analyzer (YOKOGAWA AQ6370). Actually in the experiments, the smallest waist diameter for the proposed SPTS fibre structure achieved was circa $20 \mu \mathrm{m}$. It was also found that the sensitivity of the SPTS fibrerefractometer increases as the waist diameter of the periodical tapers decreases. However the mechanical stability of the SPTS fibrerefractometer with thinner waist diameters during the RI measurements was poor, also that structure is usually associated with high losses. Thus in this works, we adopted a waist diameter of $90 \mu \mathrm{m}$ as a reasonable compromise size, to provide mechanical stability and acceptable RI sensitivity.

Fig. 7 presents the measured transmission spectra of the SPTS fiber structures before and after the tapering processes for different numbers of tapers. From Fig. 7 it is clear that the attenuation induced by each taper decreases gradually with an increase in the number of tapers; for example the first 5 periodical tapers induced an attenuation of $10 \mathrm{~dB}$ whereas the following 5 tapers induced an average attenuation of $5 \mathrm{~dB}$. As shown in Fig. 7, total attenuation of circa $25 \mathrm{~dB}$ for 20 periodical tapers was induced over the whole measured wavelength range from 1300 to $1700 \mathrm{~nm}$ after the 20th taper was created. The total attenuation induced by tapers increases only marginally if more tapers are fabricated in the fiber. The figure also shows that when the number of tapers increases, the intensity of multimode interference increases correspondingly, demonstrating more interference dips over the wavelength range. 


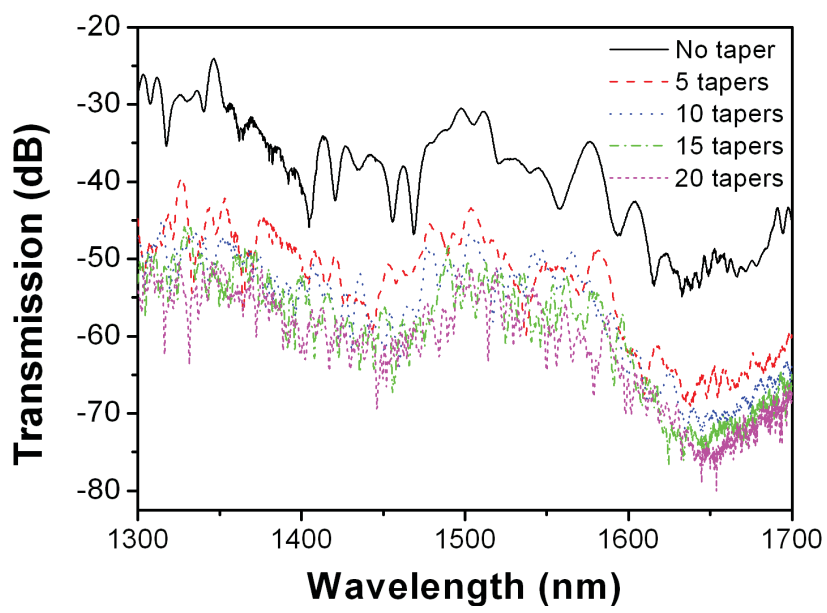

Fig. 7. Transmission spectra of the SMF28-small core fiber-SMF28 fiber without and with periodical tapers during the fabrication process.

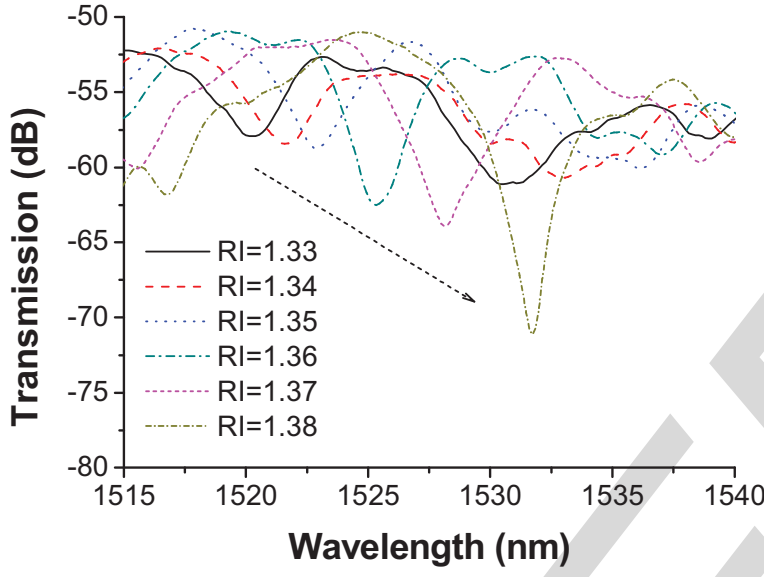

(a)

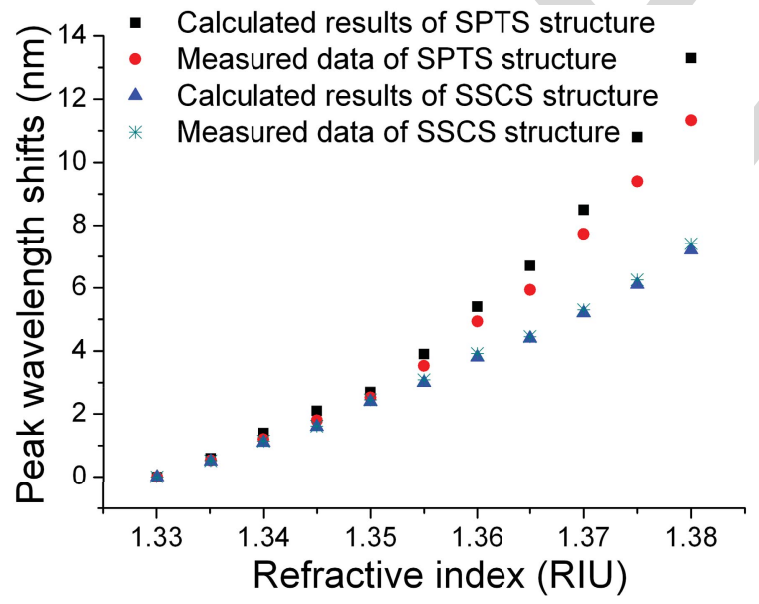

(b)

Fig. 8. (a) Measured spectral response at different surrounding RIs. (b) Calculated and measured peak wavelength shift of SPTS structure versus surrounding RI. For comparison, both calculated and measured data for SSCS structure-based refractometer are also presented.

An investigation of the refractive index sensing capability
as performed at room temperature $\left(\sim 25^{\circ} \mathrm{C}\right)$ with a series

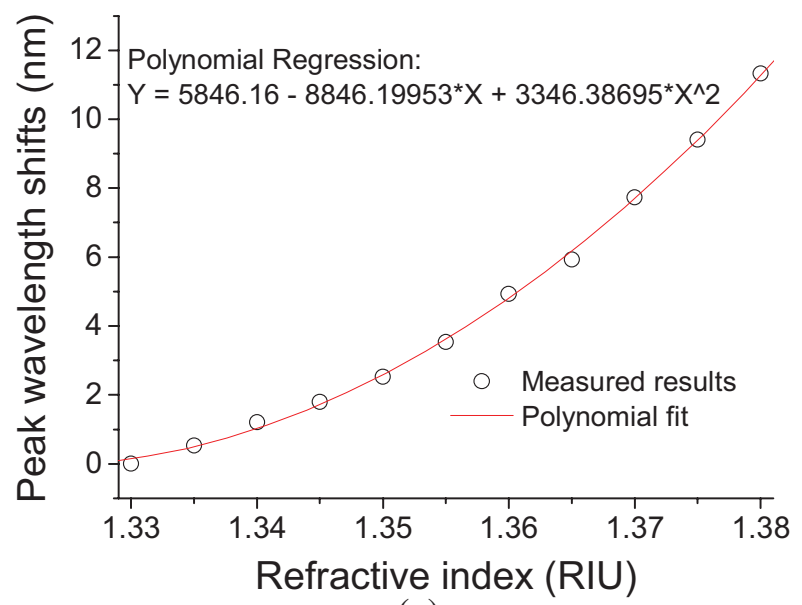

(a)

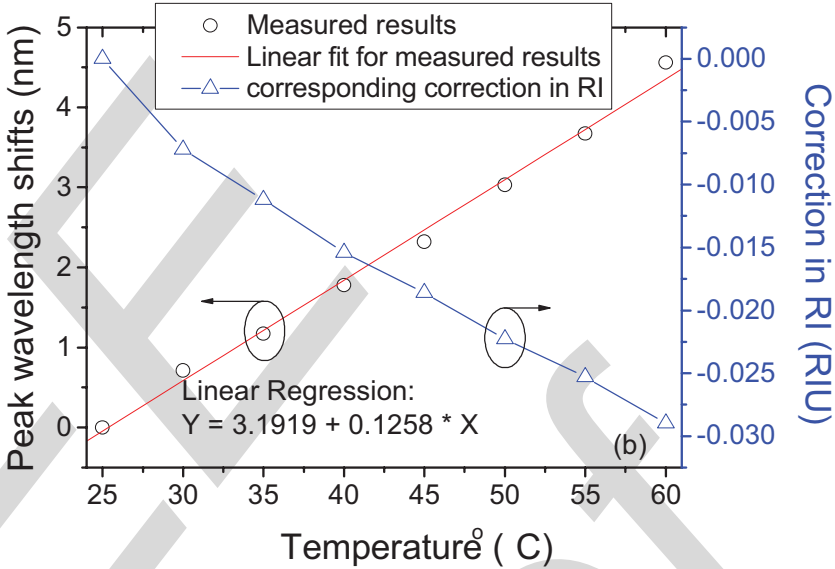

(b)

Fig. 9. (a) Measured peak wavelength shift as a function of RI and its polynomial fit. (b) Peak wavelength shift and corresponding correction in refractive index as a function of temperature variation.

of RI liquids $(1.33 \sim 1.38$ with an interval of 0.005$)$. The RI liquids were placed so as to cover the entire length of the SPTS fiber using a dropper. The measured peak wavelength shifts in the wavelength range near $1520-1535 \mathrm{~nm}$ are plotted in Fig. 8(b) as a function of RI, which shows a general agreement with the calculated results using a WA-BPM. For comparison, both calculated and measured data for an SSCS structure based refractometer are also presented in the figure. As expected from the theoretical analysis presented in Sec. 2, the curve follows an essentially exponential distribution with an average sensitivity of $226.6 \mathrm{~nm} / \mathrm{RIU}$ over an RI range of $1.33 \sim 1.38$. A maximum sensitivity of $383 \mathrm{~nm} / \mathrm{RIU}$ is achieved for an $\mathrm{RI} \sim 1.38$, resulting in a resolvable index change of $4.41 \times 10^{-5}$ for a resolvable wavelength change of $0.01 \mathrm{~nm}$, which is considerably better than the experimental results presented in Ref. [16].

\section{Temperature Dependences of the Proposed Fiber REFRACTOMETER}

In real-world fiber optic sensing applications, temperature effects are well known to have a significant influence on the properties of a fiber optic sensor, through both thermooptic and thermal expansion effects in the fiber materials. Therefore, it 
is necessary to investigate the temperature-dependent behavior of the proposed fiber refractive index sensor. For this purpose the SPTS fiber sample was placed on a temperature-controlled heating stage. A reference ratio response of the system was obtained at room temperature $\left(25^{\circ} \mathrm{C}\right)$. The variation in the spectral response from the reference response at circa $25{ }^{\circ} \mathrm{C}$ was measured for a temperature range from $25{ }^{\circ} \mathrm{C}$ to $\sim 60{ }^{\circ} \mathrm{C}$ with an interval of $5{ }^{\circ} \mathrm{C}$. The dependence of the peak wavelength shift on temperature is shown in Figure 9(b). The measured average slope of the resonance peak shift is $0.13 \mathrm{~nm} /{ }^{\circ} \mathrm{C}$. This result shows that the sensor has a rather strong temperature dependence. This temperature-dependent resonance peak shift is expected and it has been discussed in previously published work [19], [20]. However since the resonant peak monotonically redshifts with temperature, it is possible to apply a correction factor to mitigate the temperature induced errors. To verify this, the required temperature correction for the ratio response, which is effectively a correction factor for the refractive index, is calculated using the polynomial fit presented in Figure 9(a) and shown in Figure 9(b) for different temperatures with an interval of $5{ }^{\circ} \mathrm{C}$ in the range from 25 to $60{ }^{\circ} \mathrm{C}$.

\section{CONCLUSION}

In conclusion, an all-fiber refractive index sensor with a structure consisting of a series of periodical tapers is proposed and investigated experimentally. A maximum sensitivity of $338 \mathrm{~nm} / \mathrm{RIU}$ and an average sensitivity of $226.6 \mathrm{~nm} / \mathrm{RIU}$ (RI Unit) have been experimentally achieved at RI range from 1.33 to 1.38 with a $\sim 90 \mu \mathrm{m}$ periodically tapered waist diameter. The temperature-induced variations in refractive index measurements and the corresponding correction method have been investigated and presented in this article. Further optimization of the fiber sensor geometry will result in a more compact refractometric sensor device with improved performance.

\section{REFERENCES}

[1] T. Guo, H. Y. Tam, P. A. Krug, and J. Albert, "Reflective tilted fiber Bragg grating refractometer based on strong cladding to core recoupling," Opt. Express, vol. 17, no. 7, pp. 5736-5742, 2009.

[2] O. Frazao, T. Martynkien, J. M. Baptista, J. L. Santos, W. Urbanczyk, and J. Wojcik, "Optical refractometer based on a birefringent Bragg grating written in an H-shaped fiber," Opt. Lett., vol. 34, no. 1, pp. 76-78, 2009.

[3] M. Han, F. W. Guo, and Y. F. Lu, "Optical fiber refractometer based on cladding-mode Bragg grating," Opt. Lett., vol. 35, no. 3, pp. 399-401, 2010.

[4] L. Rindorf and O. Bang, "Highly sensitive refractometer with a photoniccrystal-fiber long-period grating," Opt. Lett., vol. 33, no. 6, pp. 563-565, 2008.

[5] M. Jiang, A. Zhang, Y. Wang, H. Tam, and S. He, "Fabrication of a compact reflective long-period grating sensor with a cladding-modeselective fiber end-face mirror," Opt. Exp., vol. 17, no. 20, pp. 1797617982, 2009.

[6] T. Zhu, Y. Song, Y. Rao, and Y. Zhu, "Highly sensitive optical refractometer based on edge-written ultralong-period fiber grating formed by periodic grooves," IEEE Sensors J., vol. 9, no. 6, pp. 678-681, Jun. 2009.

[7] H. M. Liang, H. Miranto, N. Granqvist, J. W. Sadowski, T. Viitala, B. C. Wang, and M. Yliperttula, "Surface plasmon resonance instrument as a refractometer for liquids and ultrathin films," Senosrs Actuat. B, vol. 149, no. 1, pp. 212-220, 2010.
[8] J. Castillo, H. Gutierrez, J. Chirinos, and J. C. Perez, "Surface plasmon resonance device with imaging processing detector for refractive index measurements," Opt. Commun., vol. 283, no. 20, pp. 3926-3930, 2010.

[9] J. Y. Lee and S. K. Tsai, "Measurement of refractive index variation of liquids by surface plasmon resonance and wavelength-modulated heterodyne interferometry," Opt. Commun., vol. 284, no. 4, pp. 925929, 2011.

[10] F. Xu and G. Brambilla, "Demonstration of a refractometric sensor based on optical microfiber coil resonator," Appl. Phys. Lett., vol. 92, no. 10, pp. 101126-1-101126-3, 2008.

[11] Y. H. Tai and P. K. Wei, "Sensitive liquid refractive index sensors using tapered optical fiber tips," Opt. Lett., vol. 35, no. 7, pp. 944-946, 2010.

[12] J. Kou, J. Feng, Q. Wang, F. Xu, and Y. Lu, "Microfiber-probe-based ultrasmall interferometric sensor," Opt. Lett., vol. 35 , no. 13, pp. 23082310,2010

[13] J. Kou, S. Qiu, F. Xu, Y. Lu, Y. Yuan, and G. Zhao, "Miniaturized metal-dielectric-hybrid fiber tip grating for refractive index sensing," IEEE Photon. Technol. Lett., vol. 23, no. 22, pp. 1712-1714, Nov. 15, 2011.

[14] P. Wang, Y. Semenova, Q. Wu, G. Farrell, Y. Ti, and J. Zheng, "Macrobending single-mode fiber-based refractometer," Appl. Opt., vol. 48, no. 31, pp. 6044-6049, 2009.

[15] Q. Wang and G. Farrell, "All-fiber multimode-interference-based refractometer sensor: Proposal and design," Opt. Lett., vol. 31, no. 3, pp. 317-319, 2006.

[16] Q. Wu, Y. Semenova, P. Wang, and G. Farrell, "A comprehensive analysis verified by experiment of a refractometer based on an SMF28-smallcore singlemode fiber (SCSMF)-SMF28 fiber structure," J. Opt., vol. 13, no. 12 , p. 125401,2011

[17] D. D. Davis, T. K. Gaylord, E. N. Glytsis, S. G. Kosinski, S. C. Mettler, and A. M. Vengsarkar, "Long-period fibre grating fabrication with focused $\mathrm{CO}_{2}$ laser pulses," Electron. Lett., vol. 34, no. 3, pp. 302303, 1998.

[18] P. Wang, G. Brambilla, M. Ding, Y. Semenova, Q. Wu, and G. Farrell, "A high sensitivity, evanescent field refractometric sensor based on tapered multimode fiber interference," Opt. Lett., vol. 36, no. 12, pp. 2233-2235, 2011.

[19] R. Jha, J. Villatoro, G. Badenes, and V. Pruneri, "Refractometry based on a photonic crystal fiber interferometer," Opt. Lett., vol. 34, no. 5, pp. 617-619, 2009.

[20] H. Kim, O. Kwon, S. Lee, and Y. Han, "Polarization-dependent refractometer for discrimination of temperature and ambient refractive index," Opt. Lett., vol. 37, no. 11, pp. 1802-1804, 2012.

Pengfei Wang received the Ph.D. degree from the Photonics Research Centre (PRC), Dublin Institute of Technology, Dublin, Ireland, in 2008.

He was a Research Assistant with the Institute of Microelectronics and Microsystems, Section of Bologna, Italian National Research Council, in 2004, and then a Research Associate with the PRC, in 2009. He joined the Optoelectronics Research Centre (ORC), University of Southampton, Southampton, U.K., as an IRC Marie Curie Research Fellow, in 2010. He joined the Advanced Laser Laboratory, SPI Lasers, Southampton, as a part-time Research Fellow, in 2011. He is currently with the PRC and involved in research for the "Return phase" of the Marie Curie Fellowship that he received, collaborated with the ORC. His current research interests include nonlinear microresontors, fiber lasers, computational photonics (modeling, simulation and optimization), photonic devices, such as microfiber- and nanowire-based fiber devices, fiber optics sensors, photonic integrated circuits, liquid crystal devices, laser machining, and applications development (optical communication and optical sensing). He has authored or co-authored more than 100 articles in academic journals and international conferences. 
Yuliya Semenova received the Graduate degree from Lviv Polytechnic National University, Ukraine, and the Ph.D. degree in physics of liquid crystals from the Ukrainian Academy of Sciences, in 1992 and 1999, respectively.

She was a Researcher with the Faculty of Electrophysics, Lviv Polytechnic National University, from 1997 to 2001. Since 2001, she has been with the School of Electronic and Communications Engineering, Dublin Institute of Technology, Dublin, Ireland, where she is currently a Lecturer and a Senior Researcher with the Photonics Research Center. She has authored or coauthored more than 100 journal and conference papers. Her current research interests include liquid crystals, photonics, and fiber optic sensing.

Qiang Wu received the B.S. degree from Beijing Normal University, Beijing, China and the Ph.D. degree from the Beijing University of Posts \& Telecommunications, Beijing, in 1996 and 2004, respectively.

He was a Senior Research Associate with the Optoelectronics Research Centre, City University of Hong Kong, from 2004 to 2006, where he was involved in research on polymer optical waveguides. He was a Research Associate with the Applied Optics and Photonics Group, Heriot-Watt University, from 2006 to 2008, where he was involved in research on laser joining in micromanufacturing. He is currently a Stokes Lecturer with the Photonics Research Centre, Dublin Institute of Technology, Dublin, Ireland. His current research interests include the design and fabrication of fiber Bragg grating devices for sensing, singlemode-multimode-singlemdoe fiber structures for novel fiber optical couplers and sensors, and surface plasmon resonant.

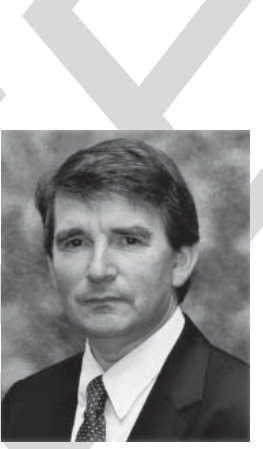

Gerald Farrell received the Degree (Hons.) in electronic engineering from University College Dublin, Dublin, Ireland, in 1979, and the Ph.D. degree from Trinity College Dublin, Dublin, with research on all-optical synchronization using self-pulsating laser diodes.

$\mathrm{He}$ is the Founder and the Director of the Photonics Research Centre, Dublin Institute of Technology (DIT), Dublin, Ireland. For many years, he was a Communications Systems Design Engineer and was involved in development of optical fiber transmission systems before joining the DIT. He has been the Head of the School of Electronic and Communications Engineering, DIT, since 2001. He was the Director of the startup company PX Instrument Technology, from 1997 to 2003, and was involved in research on optical fiber system test and measurement systems. He has authored or co-authored more than 190 publications in photonics. His current research interests incude optical sensing, particularly FBG interrogation systems, modeling and applications of fiber bend loss to optical sensing, SMS and other fiber structures for sensing applications, PCF sensors for environmental sensing and for sensing strain in composite materials and medical devices, LC infiltrated PCF sensors, and microfiber and nanowire sensors for biosensing.

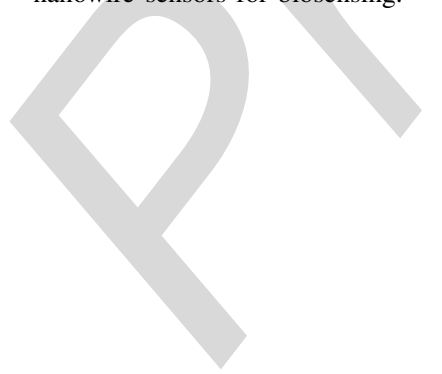

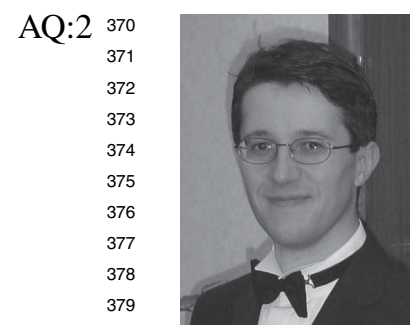

Gilberto Brambilla received the M.Sc. degree in engineering (cum laude) from the Politecnico di Milano, Milan, Italy, and the Ph.D. degree in optoelectronics from the Optoelectronics Research Centre, University of Southampton, Southampton, U.K., in 1996 and 2002, respectively.

He is currently a Principle Research Fellow with the University of Southampton. His current interests include fabrication of devices based on optical fiber nanowires, couplers and tapers, study of photosensitivity in silica-based glasses, manufacture of Bragg gratings for sensors and investigation of their thermal stability, and fabrication of fiber sensors for X-rays and nuclear radiations. He has authored or co-authored more than 200 articles in academic journals and international conferences, and three book chapters. He holds three patents. He has given more than 25 invited talks and tutorials over five continents.
Ming Ding received the B.Sc. (Hons.) degree from Beijing Jiaotong University, China. She is currently pursuing the Ph.D. degree at the Optical Fibre Nanowires and Related Devices Group, Optoelectronics Research Centre, University of Southampton, Southampton, U.K..

Her current research interests include light confinement with nanostructured optical fiber taper, micro- and nanoresonators, micro- and nanofiber Bragg grating, and micro- and nanofiber sensors.
Timothy Lee received the M.Eng. degree in electronic engineering from the University of Southampton, Southampton, U.K., in 2009, where he undertook an internship with the Nano Research Group.

He joined the Optoelectronics Research Centre University of Southampton, in 2009. His current research interests include the nonlinear behavior and polarization-dependent effects of microfibers and their resonators. 


\section{AUTHOR QUERIES}

AQ:1 = Please specify the major field of the Ph.D. degree.

AQ:2 = Please specify the major field of the M.Sc. degree.

AQ:3 = Please specify the major field of the B.Sc. degree.

AQ:4 = Please specify the which degree and major field.

AQ:5 = Please specify the major field of the B.S. and Ph.D. degrees.

AQ:6 = Please specify the year of completion of the Ph.D. degree.

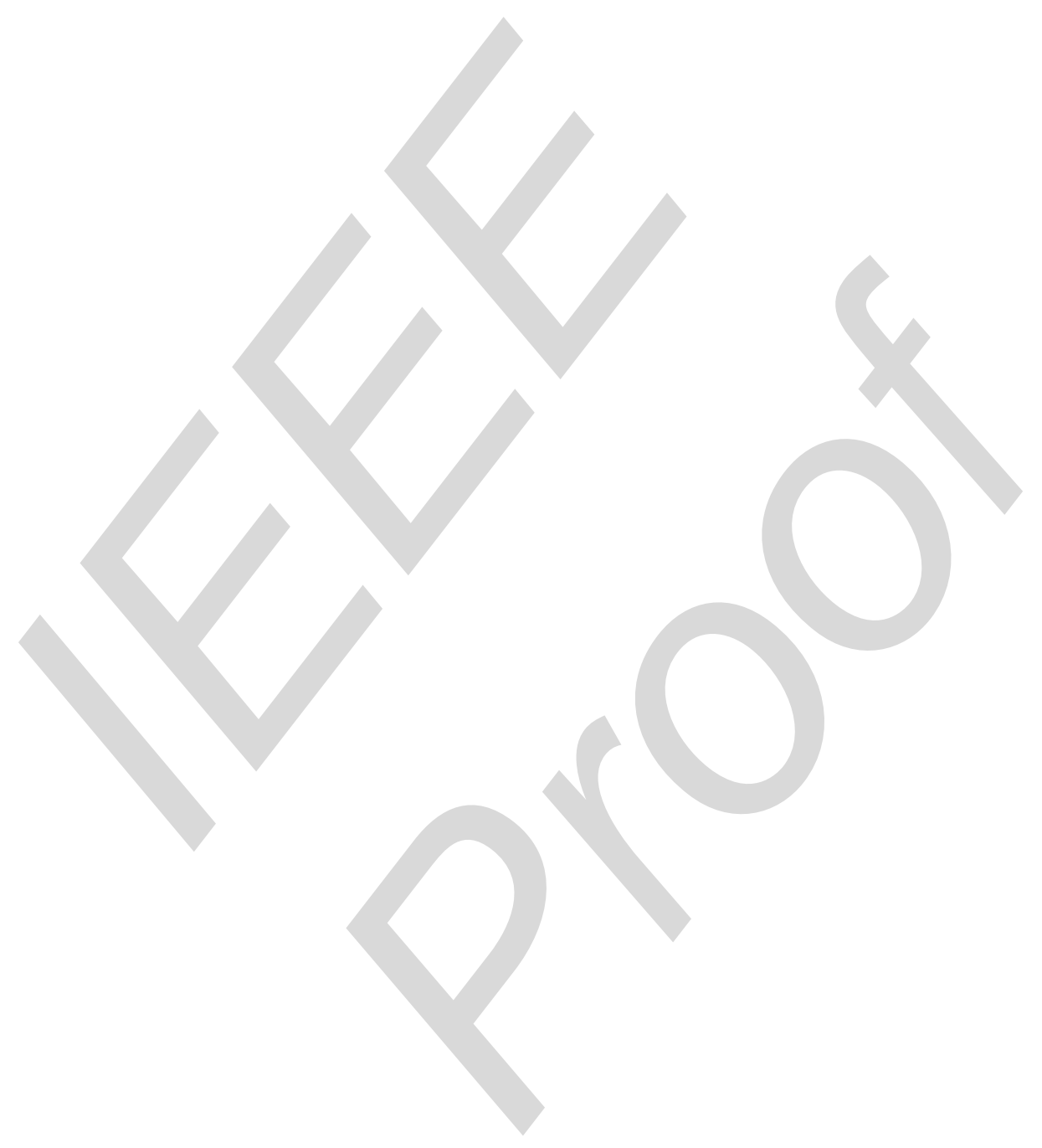




\title{
Enhanced Refractometer Based on Periodically Tapered Small Core Singlemode Fiber
}

\author{
Pengfei Wang, Gilberto Brambilla, Ming Ding, Timothy Lee, Lin Bo, \\ Yuliya Semenova, Qiang Wu, and Gerald Farrell
}

\begin{abstract}
An all-fiber refractive index (RI) sensor with a simple configuration of periodical tapers is proposed and investigated experimentally. The proposed fiber RI sensor consists of a small core fiber sandwiched between two standard singlemode fibers, with tapers periodically fabricated along the small core fiber using a focused $\mathrm{CO}_{2}$ laser beam. Such a structure can be used for RI sensing by measuring the dip wavelength shift of the multimode interference within the small core fiber cladding. An average sensitivity of $226.6 \mathrm{~nm} / \mathrm{RIU}$ (RI Unit) has been experimentally achieved in the RI range from 1.33 to 1.38 . The refractometer is sensitive to temperature and an experimental investigation of this sensitivity is presented. It is found that the peak shift response has a linear variation with temperature; therefore, temperature dependence can be mitigated by a suitable RI correction process. The proposed RI sensor benefits from simplicity and low cost and achieves a competitive sensitivity compared with other existing fiber-optic sensors.
\end{abstract}

Index Terms-Fiber gratings, fiber optics, optical fiber device, optical fiber sensors.

\section{INTRODUCTION}

O PTICAL fiber refractive index (RI) sensors offer advantages such as immunity to electromagnetic interference, high sensitivity, fast response, small size and ease of fabrication. To date several types of optical fiber refractometers have been proposed for applications in growth areas for RI sensing [1]-[12]. The most common approaches are refractometers based on on a fiber Bragg grating [1]-[3], long period grating [4]-[6], surface plasmon [7]-[9], tapered microfiber [10]-[13],

Manuscript received May 23, 2012; revised August 11, 2012; accepted August 27, 2012. This work was supported in part by the National Access Program, Tyndall National Institute, Cork, Ireland. The work of P. Wang was supported by the Irish Research Council, co-funded by the MarieCurie Actions under FP7. The work of G. Brambilla was supported by the Royal Society (London) for his research fellowship. The work of Q. Wu was supported by the Science Foundation Ireland under Grant 07/SK/I1200, Grant 11/TIDA/B2051, Grant 07/SK/I1200-STTF11, and Grant 07/SK/I1200ISTTF11. The associate editor coordinating the review of this paper and approving it for publication was Prof. Michael Schöning.

P. Wang is with the Optoelectronics Research Centre, University of Southampton, Southampton SO17 1BJ, U.K., and also with the Photonic Research Centre, Dublin Institute of Technology, Dublin 8, Ireland (e-mail: pw3y09@orc.soton.ac.uk).

G. Brambilla, M. Ding, and T. Lee are with the Optoelectronics Research Centre, University of Southampton, Southampton SO17 1BJ, U.K. (e-mail: gb2@orc.soton.ac.uk; md20d09@orc.soton.ac.uk; t1305@orc.soton.ac.uk).

L. Bo, Y. Semenova, Q. Wu, and G. Farrell are with the Photonic Research Centre, Dublin Institute of Technology, Dublin 8, Ireland (e-mail: bo.lin@mydit.ie; yuliya.semenova@dit.ie; qiang.wu@dit.ie; gerald.farrell@ dit.ie).

Color versions of one or more of the figures in this paper are available online at http://ieeexplore.ieee.org.

Digital Object Identifier 10.1109/JSEN.2012.2216865

bent fiber [14] and a singlemode fiber (SMF)-multimode fiber (MMF)-singlemode fiber [15]. As an alternative to these existing fiber refractometers, an SMF28 -small core -SMF28 (SSCS) fiber structure based fiber refractometer [16] has been proposed recently featuring a high sensitivity of $102 \mathrm{~nm} / \mathrm{RIU}$ at an RI $=1.324$. With respect to the other techniques, an SSCS provides a reliable high sensitivity sensor at low cost.

For RI sensing, the SSCS structure proposed in Ref. [16] needs strong multimode interference between the fiber cladding modes, thus the diameter of the cladding of the small core fiber in an SSCS structure should be small in order to achieve high sensitivity. Experimentally, an effective approach is to employ a small core fiber with a reduced cladding diameter achieved by etching with hydrofluoric acid, but this increases the fabrication difficulty and the induced surface roughness may lead to a significant discrepancy between experimental results and theoretical design [14]. Alternatively, fiber tapering can replace the chemical etching process; since light guided in a tapered fiber has a significant fraction of power propagating as an evanescent wave, the effective index of the guided mode is affected by the surrounding medium RI. It is well known that the fraction of power in the form of evanescent field, and thus its sensitivity to environmental changes, increases for smaller taper diameters.

Since Davis et al. [17] reported the first example of long period fibre grating (LPFG) written by $\mathrm{CO}_{2}$ laser irradiation in a conventional glass fiber in 1998, this method has been used to manufacture a variety of devices and sensors. Compared with the LPFG fabricated by a UV-laser exposure technique, the $\mathrm{CO}_{2}$ laser irradiation technique is much more flexible and low cost because no photosensitivity or sensitization such as hydrogen loading are needed. Moreover the $\mathrm{CO}_{2}$ laser irradiation process can be controlled to generate complicated grating profiles via the well-known point-to-point technique without the use of any expensive masks. Therefore this technique can be used to write LPFGs in almost all types of fibers including pure-silica photonic crystal fibers. In this paper, we report the experimental demonstration of a novel high sensitivity refractometric sensor based on multimodal interference in a singlemode-periodically tapered small core fiber-singlemode fiber structure (SPTS) capable of improving the sensitivity using a series of microtapers [18]. The small core fiber used in the experiments was a commercial fiber (Nufern S405 HP) with a core diameter of circa $2.5 \mu \mathrm{m}$ and a cut-off wavelength of $365 \mathrm{~nm}$. The schematic configuration of the SSCS used as the starting point to fabricate the SPTS used in the experiments 


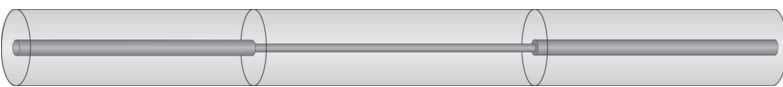

Fig. 1. Schematic of the SMF28-small core fiber-SMF28 fiber structure.

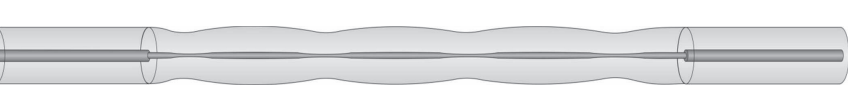

Fig. 2. Schematic of the SMF28-small core fiber with periodical tapersSMF28 fiber structure.

is shown in Fig. 1. Light propagation in such an SSCS fiber section has been simulated and analysed using an eigenmode analytical method presented in Ref. [16]. During the experimental verification, it was found that the power of the core mode guided in such an SSCS structure is low $(\sim-25 \mathrm{~dB})$ and can thus be ignored in the theoretical model, this supports the fact that only the multimode interference between the cladding modes is essential for the device to work and should be considered.

Ref. [18] has shown that if the multimode section is tapered in a conventional singlemode-multimode-singlemode (SMS) structure, then strong mode interference occurs within the tapered MMF section due to the focusing effects of the input section to the taper. Within the tapered MMF section, the excited modes of LPOm in the MMF core will be partly coupled to the high-order cladding modes at the beginning of the fiber taper region and this increases the fraction of power in the evanescent field within the region of MMF cladding. This phenomenon offers a possibility to increase the intensity of multimode interference of the cladding modes in an SSCS using a tapered structure. Furthermore, by using several concatenated tapers, a periodic taper structure is created as shown in Fig. 2, consisting of an input SMF, a periodically tapered small core fiber section and an output SMF. Such an SPTS structure offers the potential to achieve a higher sensitivity than a single taper alone due to the increased cladding mode interference induced by the multiple focusing effects of the input section of each of the tapers.

\section{TheORETICAL ANALYSis}

In our recent published work, a comprehensive theoretical analysis for an SSCS fiber structure has been presented using an eigenmode analytical method. The SSCS fiber with surrounding RI liquid can be treated as a three layer structure without a fundamental core mode guided in the core of the small core fiber. The good agreement between the calculated and measured results has shown that the theoretical eigenmode analytical method employed is an effective way to predict the performance of an SSCS fiber refractometer. Since in practice there is no fundamental core mode guided in the small core fiber, therefore the three layer fiber structure (core-cladding-surrounding RI) can be simplified as a two layer fiber structure, namely a cladding-surrounding RI structure. This offers a possibility to simulate the light propagation in the SSCS fiber structure using a wide angle-beam propagation method (WA-BPM) with cylindrical coordinates using a Padé $(3,3)$ approximate operator and perfectly matched layer (PML) boundary conditions which has been presented in the

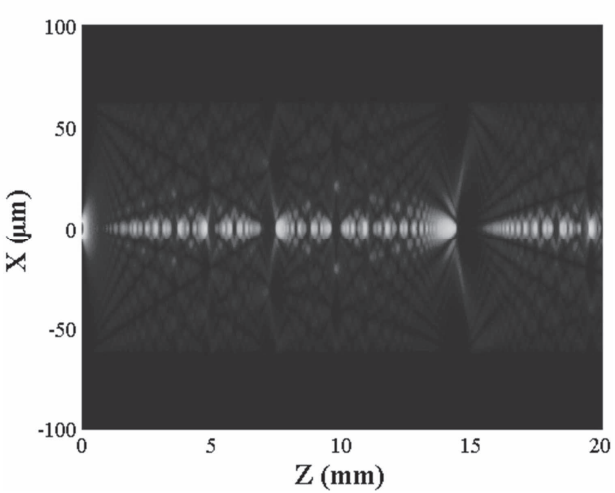

(a)

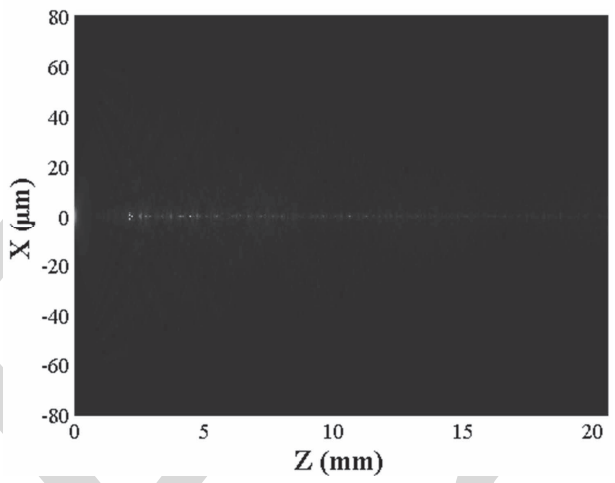

(b)

Fig. 3. Calculated amplitude of optical fields at a wavelength of $1550 \mathrm{~nm}$ using a WA-BPM for (a) SSCS fiber sandwiched between two standard SMFs, and (b) SPTS fiber with 28 microtapers with a waist diameter of $90 \mu \mathrm{m}$, sandwiched between two standard SMFs.

Ref. [18]. To prove the foundation of this approach, an alcoholbased graphite adhesive with high absorption coefficient was applied to the $2 \mathrm{~cm}$ long surface of the small core fiber sandwiched between two SMFs. Light transmitted in the SSCS fiber structure experienced $\mathrm{a} \sim 25 \mathrm{~dB}$ attenuation, hence verifying that almost all of the light is actually transmitted in the cladding of the small core fiber.

The calculated amplitudes of the optical fields of both SSCS and SPTS fiber structures are presented in Fig. 3(a) and (b) respectively. Fig. 3(a) shows for reference the expected optical field at a wavelength of $1550 \mathrm{~nm}$ for an SSCS section, while Fig. 3(b) shows that weakly confinement occurs within the SPTS section due to the reduced size of fiber core diameter within the microtapers: at long wavelengths, beyond the cutoff wavelength, such as $1550 \mathrm{~nm}$, the core mode expands out into the surrounding silica cladding as the core diameter reduces. This results in a more efficient coupling with the fiber cladding modes compared with the SSCS fiber structure, which explains the non-adiabatic and highly lossy behavior of the SPTS fiber structure. Compared with the SSCS structure shown in Fig. 3(a), the optical power propagating through the fiber core of the SPTS structure (fig. 3(b)) is very low because of the enhanced multimode interference and weak confinement of the fiber core mode.

The peak wavelength shifts of both SSCS and SPTS fiber structures as a function of surrounding RI are calculated using a WA-BPM. The simulated results in figure 4 confirm that 


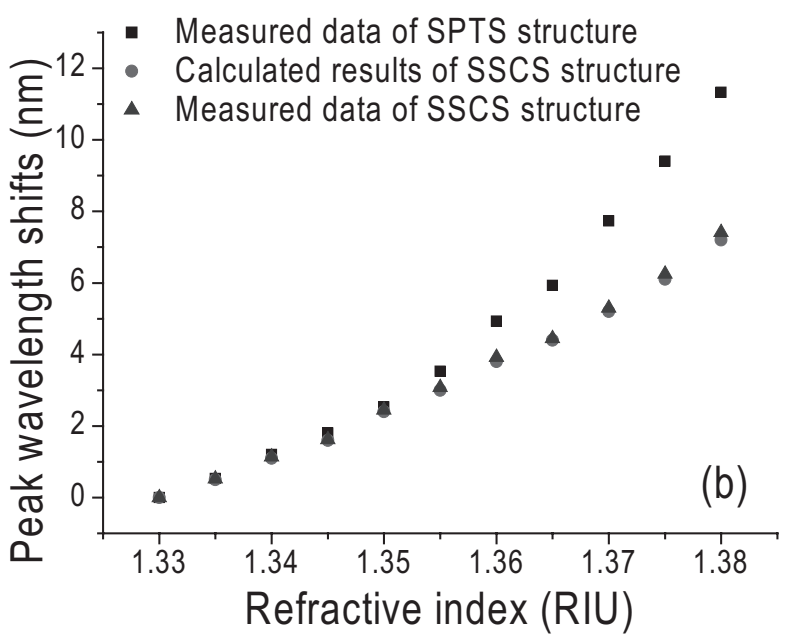

Fig. 4. Calculated peak wavelength shifts of the SSCS and SPTS fiber structures as a function of surrounding RI.

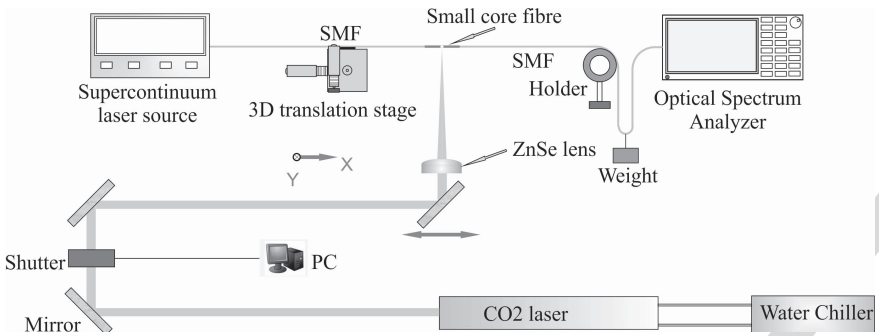

Fig. 5. Schematic of the fabrication and experimental setup.

as the surrounding RI increases the central wavelengths of both SSCS and SPTS fiber structures increase monotonically in an exponential fashion. The peak wavelength shift of the SPTS fiber structure is much higher than that of the SSCS fiber structure, confirming that the refractometer with a SPTS structure has a higher sensitivity than that with a SSCS fiber structure.

\section{FABRiCATION OF THE SPTS FibER StRUCTURE}

The SSCS fiber sample used as the starting point for the SPTS was fabricated from a $21 \mathrm{~mm}$ length of Nufern S405 HP step index SMF which was stripped, cleaved and then spliced between two standard SMF28 fibers. As shown in the fabrication setup in Fig. 5, a $\mathrm{CO}_{2}$ laser (SYNRAD, Model: $48-2 \mathrm{KWL}$, with a maximum power 30 Watts at a wavelength of $10.6 \mu \mathrm{m}$ ) was employed to fabricate the tapered fiber. A ZnSe cylindrical lens with a focal length of $254 \pm 0.5 \%$ $\mathrm{mm}$ focused the $\mathrm{CO}_{2}$ laser beam to $\sim 200 \mu \mathrm{m}$. Beam movement was achieved using gold-coated mirrors on a motorized translation stage. A Labview program controlled the shutter opening and therefore the laser exposure time. One 3D translation stage was used to adjust the heating position within the small core fiber section, while a weight $(\sim 3.7 \mathrm{~g})$ was used to apply a constant tension to the SMF end of the SSCS structure. The small core fiber was exposed to the $\mathrm{CO}_{2}$ laser beam with an output power of $10.5 \mathrm{~W}$ for $120 \mathrm{~s}$ and tapering occurred because of the simultaneous localized heating of the fiber and tension applied to the end

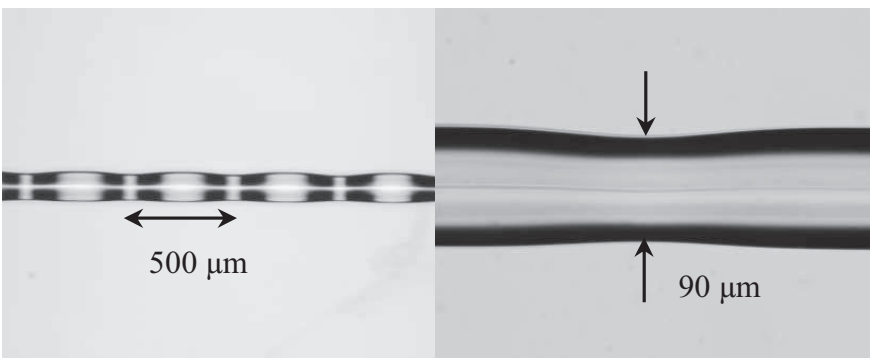

Fig. 6. Images of the small core fiber with periodic microtapers. The image was obtained with a microscope (Nikon Eclipse LV100) with a $5 \times$ (top) and $20 \times$ (bottom) objective, respectively.

of the SMF. Then the laser beam was translated a distance of $400 \mu \mathrm{m}$ along the fiber axis to irradiate and taper another segment of the fiber. This tapering process was repeated up to 20 times. Consequently, periodic tapers were created on the small core fiber, as shown in Fig. 6(a). Although the translation distance of the laser beam is $400 \mu \mathrm{m}$ along the fiber axis, the actual period of the tapers is circa $500 \mu \mathrm{m}$ due to the elongation of the fiber induced by tapering. The tensioning weight used during fiber processing was removed for subsequent refractive index measurements, with the fiber sample mounted instead on a "U" shaped stage.The detail of one of the resulting tapers is shown in Fig. 6(b), the waist diameter is circa $90 \mu \mathrm{m}$. The transmission spectra of the SPTS fiber structure were monitored and recorded during fabrication using a Supercontinuum source (Fianium Ltd, U.K.), which delivered $50 \mathrm{~nJ}$ light pulses over a broad range of wavelengths (450-1800 nm), and a high resolution (20 pm) optical spectrum analyzer (YOKOGAWA AQ6370). Actually in the experiments, the smallest waist diameter for the proposed SPTS fibre structure achieved was circa $20 \mu \mathrm{m}$. It was also found that the sensitivity of the SPTS fibrerefractometer increases as the waist diameter of the periodical tapers decreases. However the mechanical stability of the SPTS fibrerefractometer with thinner waist diameters during the RI measurements was poor, also that structure is usually associated with high losses. Thus in this works, we adopted a waist diameter of $90 \mu \mathrm{m}$ as a reasonable compromise size, to provide mechanical stability and acceptable RI sensitivity.

Fig. 7 presents the measured transmission spectra of the SPTS fiber structures before and after the tapering processes for different numbers of tapers. From Fig. 7 it is clear that the attenuation induced by each taper decreases gradually with an increase in the number of tapers; for example the first 5 periodical tapers induced an attenuation of $10 \mathrm{~dB}$ whereas the following 5 tapers induced an average attenuation of $5 \mathrm{~dB}$. As shown in Fig. 7, total attenuation of circa $25 \mathrm{~dB}$ for 20 periodical tapers was induced over the whole measured wavelength range from 1300 to $1700 \mathrm{~nm}$ after the 20th taper was created. The total attenuation induced by tapers increases only marginally if more tapers are fabricated in the fiber. The figure also shows that when the number of tapers increases, the intensity of multimode interference increases correspondingly, demonstrating more interference dips over the wavelength range. 


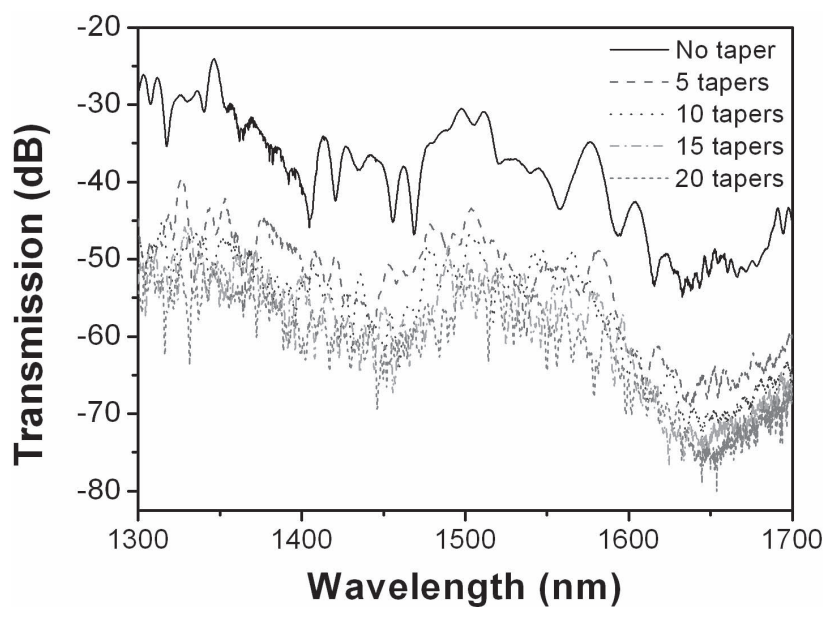

Fig. 7. Transmission spectra of the SMF28-small core fiber-SMF28 fiber without and with periodical tapers during the fabrication process.

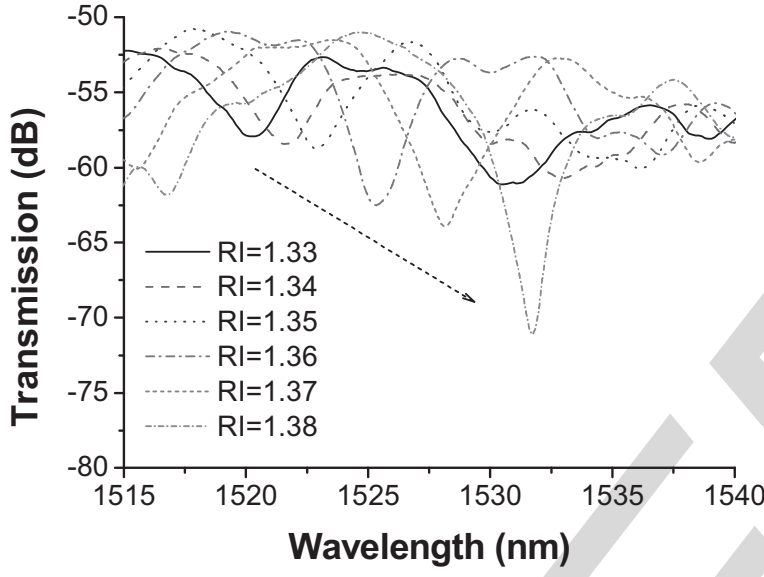

(a)

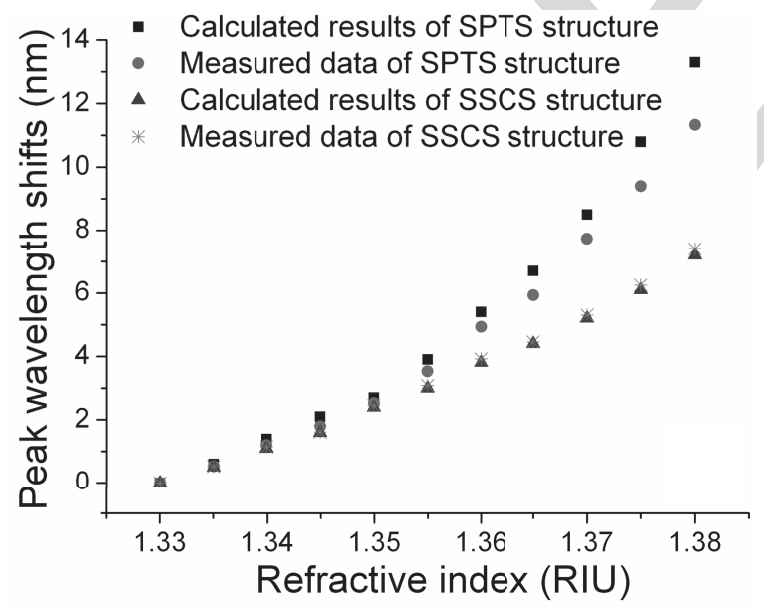

(b)

Fig. 8. (a) Measured spectral response at different surrounding RIs. (b) Calculated and measured peak wavelength shift of SPTS structure versus surrounding RI. For comparison, both calculated and measured data for SSCS structure-based refractometer are also presented.

An investigation of the refractive index sensing capability
as performed at room temperature $\left(\sim 25^{\circ} \mathrm{C}\right)$ with a series

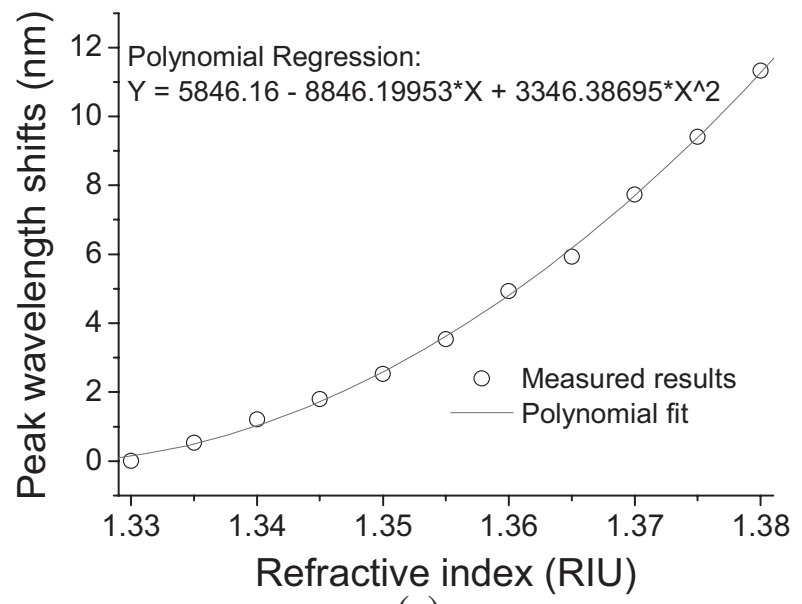

(a)

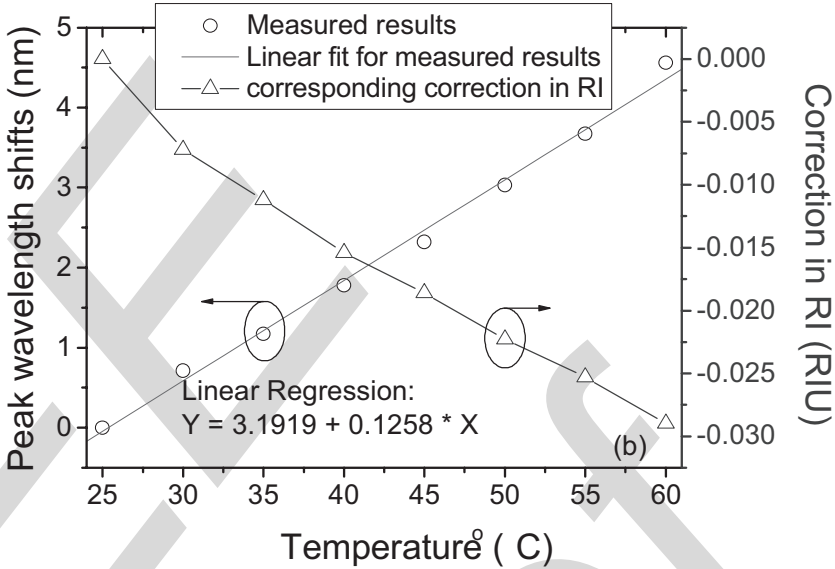

(b)

Fig. 9. (a) Measured peak wavelength shift as a function of RI and its polynomial fit. (b) Peak wavelength shift and corresponding correction in refractive index as a function of temperature variation.

of RI liquids $(1.33 \sim 1.38$ with an interval of 0.005$)$. The RI liquids were placed so as to cover the entire length of the SPTS fiber using a dropper. The measured peak wavelength shifts in the wavelength range near $1520-1535 \mathrm{~nm}$ are plotted in Fig. 8(b) as a function of RI, which shows a general agreement with the calculated results using a WA-BPM. For comparison, both calculated and measured data for an SSCS structure based refractometer are also presented in the figure. As expected from the theoretical analysis presented in Sec. 2, the curve follows an essentially exponential distribution with an average sensitivity of $226.6 \mathrm{~nm} / \mathrm{RIU}$ over an RI range of $1.33 \sim 1.38$. A maximum sensitivity of $383 \mathrm{~nm} / \mathrm{RIU}$ is achieved for an $\mathrm{RI} \sim 1.38$, resulting in a resolvable index change of $4.41 \times 10^{-5}$ for a resolvable wavelength change of $0.01 \mathrm{~nm}$, which is considerably better than the experimental results presented in Ref. [16].

\section{Temperature Dependences of the PROPOSED FIBER REFRACTOMETER}

In real-world fiber optic sensing applications, temperature effects are well known to have a significant influence on the properties of a fiber optic sensor, through both thermooptic and thermal expansion effects in the fiber materials. Therefore, it 
is necessary to investigate the temperature-dependent behavior of the proposed fiber refractive index sensor. For this purpose the SPTS fiber sample was placed on a temperature-controlled heating stage. A reference ratio response of the system was obtained at room temperature $\left(25^{\circ} \mathrm{C}\right)$. The variation in the spectral response from the reference response at circa $25{ }^{\circ} \mathrm{C}$ was measured for a temperature range from $25{ }^{\circ} \mathrm{C}$ to $\sim 60{ }^{\circ} \mathrm{C}$ with an interval of $5{ }^{\circ} \mathrm{C}$. The dependence of the peak wavelength shift on temperature is shown in Figure 9(b). The measured average slope of the resonance peak shift is $0.13 \mathrm{~nm} /{ }^{\circ} \mathrm{C}$. This result shows that the sensor has a rather strong temperature dependence. This temperature-dependent resonance peak shift is expected and it has been discussed in previously published work [19], [20]. However since the resonant peak monotonically redshifts with temperature, it is possible to apply a correction factor to mitigate the temperature induced errors. To verify this, the required temperature correction for the ratio response, which is effectively a correction factor for the refractive index, is calculated using the polynomial fit presented in Figure 9(a) and shown in Figure 9(b) for different temperatures with an interval of $5{ }^{\circ} \mathrm{C}$ in the range from 25 to $60{ }^{\circ} \mathrm{C}$.

\section{CONCLUSION}

In conclusion, an all-fiber refractive index sensor with a structure consisting of a series of periodical tapers is proposed and investigated experimentally. A maximum sensitivity of $338 \mathrm{~nm} / \mathrm{RIU}$ and an average sensitivity of $226.6 \mathrm{~nm} / \mathrm{RIU}$ (RI Unit) have been experimentally achieved at RI range from 1.33 to 1.38 with a $\sim 90 \mu \mathrm{m}$ periodically tapered waist diameter. The temperature-induced variations in refractive index measurements and the corresponding correction method have been investigated and presented in this article. Further optimization of the fiber sensor geometry will result in a more compact refractometric sensor device with improved performance.

\section{REFERENCES}

[1] T. Guo, H. Y. Tam, P. A. Krug, and J. Albert, "Reflective tilted fiber Bragg grating refractometer based on strong cladding to core recoupling," Opt. Express, vol. 17, no. 7, pp. 5736-5742, 2009.

[2] O. Frazao, T. Martynkien, J. M. Baptista, J. L. Santos, W. Urbanczyk, and J. Wojcik, "Optical refractometer based on a birefringent Bragg grating written in an H-shaped fiber," Opt. Lett., vol. 34, no. 1, pp. 76-78, 2009.

[3] M. Han, F. W. Guo, and Y. F. Lu, "Optical fiber refractometer based on cladding-mode Bragg grating," Opt. Lett., vol. 35, no. 3, pp. 399-401, 2010.

[4] L. Rindorf and O. Bang, "Highly sensitive refractometer with a photoniccrystal-fiber long-period grating," Opt. Lett., vol. 33, no. 6, pp. 563-565, 2008.

[5] M. Jiang, A. Zhang, Y. Wang, H. Tam, and S. He, "Fabrication of a compact reflective long-period grating sensor with a cladding-modeselective fiber end-face mirror," Opt. Exp., vol. 17, no. 20, pp. 17976 17982, 2009.

[6] T. Zhu, Y. Song, Y. Rao, and Y. Zhu, "Highly sensitive optical refractometer based on edge-written ultralong-period fiber grating formed by periodic grooves," IEEE Sensors J., vol. 9, no. 6, pp. 678-681, Jun. 2009.

[7] H. M. Liang, H. Miranto, N. Granqvist, J. W. Sadowski, T. Viitala, B. C. Wang, and M. Yliperttula, "Surface plasmon resonance instrument as a refractometer for liquids and ultrathin films," Senosrs Actuat. B, vol. 149 , no. 1, pp. 212-220, 2010.
[8] J. Castillo, H. Gutierrez, J. Chirinos, and J. C. Perez, "Surface plasmon resonance device with imaging processing detector for refractive index measurements," Opt. Commun., vol. 283, no. 20, pp. 3926-3930, 2010.

[9] J. Y. Lee and S. K. Tsai, "Measurement of refractive index variation of liquids by surface plasmon resonance and wavelength-modulated heterodyne interferometry," Opt. Commun., vol. 284, no. 4, pp. 925929, 2011.

[10] F. Xu and G. Brambilla, "Demonstration of a refractometric sensor based on optical microfiber coil resonator," Appl. Phys. Lett., vol. 92, no. 10, pp. 101126-1-101126-3, 2008.

[11] Y. H. Tai and P. K. Wei, "Sensitive liquid refractive index sensors using tapered optical fiber tips," Opt. Lett., vol. 35, no. 7, pp. 944-946, 2010.

[12] J. Kou, J. Feng, Q. Wang, F. Xu, and Y. Lu, "Microfiber-probe-based ultrasmall interferometric sensor," Opt. Lett., vol. 35, no. 13, pp. 2308 2310,2010

[13] J. Kou, S. Qiu, F. Xu, Y. Lu, Y. Yuan, and G. Zhao, "Miniaturized metal-dielectric-hybrid fiber tip grating for refractive index sensing," IEEE Photon. Technol. Lett., vol. 23, no. 22, pp. 1712-1714, Nov. 15, 2011.

[14] P. Wang, Y. Semenova, Q. Wu, G. Farrell, Y. Ti, and J. Zheng, "Macrobending single-mode fiber-based refractometer," Appl. Opt., vol. 48, no. 31, pp. 6044-6049, 2009.

[15] Q. Wang and G. Farrell, "All-fiber multimode-interference-based refractometer sensor: Proposal and design," Opt. Lett., vol. 31, no. 3, pp. 317-319, 2006.

[16] Q. Wu, Y. Semenova, P. Wang, and G. Farrell, "A comprehensive analysis verified by experiment of a refractometer based on an SMF28-smallcore singlemode fiber (SCSMF)-SMF28 fiber structure," J. Opt., vol. 13, no. 12 , p. $125401,2011$.

[17] D. D. Davis, T. K. Gaylord, E. N. Glytsis, S. G. Kosinski, S. C. Mettler, and A. M. Vengsarkar, "Long-period fibre grating fabrication with focused $\mathrm{CO}_{2}$ laser pulses," Electron. Lett., vol. 34, no. 3, pp. 302 303, 1998.

[18] P. Wang, G. Brambilla, M. Ding, Y. Semenova, Q. Wu, and G. Farrell, “A high sensitivity, evanescent field refractometric sensor based on tapered multimode fiber interference," Opt. Lett., vol. 36, no. 12, pp. 2233-2235, 2011.

[19] R. Jha, J. Villatoro, G. Badenes, and V. Pruneri, "Refractometry based on a photonic crystal fiber interferometer," Opt. Lett., vol. 34, no. 5, pp. 617-619, 2009.

[20] H. Kim, O. Kwon, S. Lee, and Y. Han, "Polarization-dependent refractometer for discrimination of temperature and ambient refractive index," Opt. Lett., vol. 37, no. 11, pp. 1802-1804, 2012.

Pengfei Wang received the Ph.D. degree from the Photonics Research Centre (PRC), Dublin Institute of Technology, Dublin, Ireland, in 2008.

He was a Research Assistant with the Institute of Microelectronics and Microsystems, Section of Bologna, Italian National Research Council, in 2004, and then a Research Associate with the PRC, in 2009. He joined the Optoelectronics Research Centre (ORC), University of Southampton, Southampton, U.K., as an IRC Marie Curie Research Fellow, in 2010. He joined the Advanced Laser Laboratory, SPI Lasers, Southampton, as a part-time Research Fellow, in 2011. He is currently with the PRC and involved in research for the "Return phase" of the Marie Curie Fellowship that he received, collaborated with the ORC. His current research interests include nonlinear microresontors, fiber lasers, computational photonics (modeling, simulation and optimization), photonic devices, such as microfiber- and nanowire-based fiber devices, fiber optics sensors, photonic integrated circuits, liquid crystal devices, laser machining, and applications development (optical communication and optical sensing). He has authored or co-authored more than 100 articles in academic journals and international conferences. 


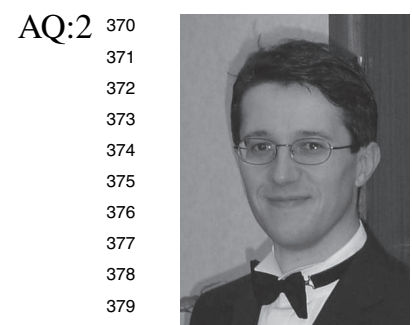

Gilberto Brambilla received the M.Sc. degree in engineering (cum laude) from the Politecnico di Milano, Milan, Italy, and the Ph.D. degree in optoelectronics from the Optoelectronics Research Centre, University of Southampton, Southampton, U.K., in 1996 and 2002, respectively.

He is currently a Principle Research Fellow with the University of Southampton. His current interests include fabrication of devices based on optical fiber nanowires, couplers and tapers, study of photosensitivity in silica-based glasses, manufacture of Bragg gratings for sensors and investigation of their thermal stability, and fabrication of fiber sensors for X-rays and nuclear radiations. He has authored or co-authored more than 200 articles in academic journals and international conferences, and three book chapters. He holds three patents. He has given more than 25 invited talks and tutorials over five continents.
Ming Ding received the B.Sc. (Hons.) degree from Beijing Jiaotong University, China. She is currently pursuing the Ph.D. degree at the Optical Fibre Nanowires and Related Devices Group, Optoelectronics Research Centre, University of Southampton, Southampton, U.K..

Her current research interests include light confinement with nanostructured optical fiber taper, micro- and nanoresonators, micro- and nanofiber Bragg grating, and micro- and nanofiber sensors.
Timothy Lee received the M.Eng. degree in electronic engineering from the University of Southampton, Southampton, U.K., in 2009, where he undertook an internship with the Nano Research Group.

He joined the Optoelectronics Research Centre University of Southampton, in 2009. His current research interests include the nonlinear behavior and polarization-dependent effects of microfibers and their resonators.
Yuliya Semenova received the Graduate degree from Lviv Polytechnic National University, Ukraine, and the Ph.D. degree in physics of liquid crystals from the Ukrainian Academy of Sciences, in 1992 and 1999, respectively.

She was a Researcher with the Faculty of Electrophysics, Lviv Polytechnic National University, from 1997 to 2001. Since 2001, she has been with the School of Electronic and Communications Engineering, Dublin Institute of Technology, Dublin, Ireland, where she is currently a Lecturer and a Senior Researcher with the Photonics Research Center. She has authored or coauthored more than 100 journal and conference papers. Her current research interests include liquid crystals, photonics, and fiber optic sensing.

Qiang Wu received the B.S. degree from Beijing Normal University, Beijing, China and the Ph.D. degree from the Beijing University of Posts \& Telecommunications, Beijing, in 1996 and 2004, respectively.

He was a Senior Research Associate with the Optoelectronics Research Centre, City University of Hong Kong, from 2004 to 2006, where he was involved in research on polymer optical waveguides. He was a Research Associate with the Applied Optics and Photonics Group, Heriot-Watt University, from 2006 to 2008 , where he was involved in research on laser joining in micromanufacturing. He is currently a Stokes Lecturer with the Photonics Research Centre, Dublin Institute of Technology, Dublin, Ireland. His current research interests include the design and fabrication of fiber Bragg grating devices for sensing, singlemode-multimode-singlemdoe fiber structures for novel fiber optical couplers and sensors, and surface plasmon resonant.

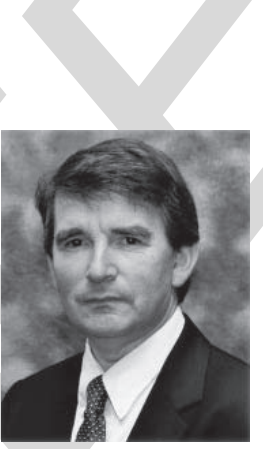

Gerald Farrell received the Degree (Hons.) in electronic engineering from University College Dublin, Dublin, Ireland, in 1979, and the Ph.D. degree from Trinity College Dublin, Dublin, with research on all-optical synchronization using self-pulsating laser diodes.

$\mathrm{He}$ is the Founder and the Director of the Photonics Research Centre, Dublin Institute of Technology (DIT), Dublin, Ireland. For many years, he was a Communications Systems Design Engineer and was involved in development of optical fiber transmission systems before joining the DIT. He has been the Head of the School of Electronic and Communications Engineering, DIT, since 2001. He was the Director of the startup company PX Instrument Technology, from 1997 to 2003, and was involved in research on optical fiber system test and measurement systems. He has authored or co-authored more than 190 publications in photonics. His current research interests incude optical sensing, particularly FBG interrogation systems, modeling and applications of fiber bend loss to optical sensing, SMS and other fiber structures for sensing applications, PCF sensors for environmental sensing and for sensing strain in composite materials and medical devices, LC infiltrated PCF sensors, and microfiber and nanowire sensors for biosensing. sensing applications. 


\section{AUTHOR QUERIES}

AQ:1 = Please specify the major field of the Ph.D. degree.

$\mathrm{AQ}: 2$ = Please specify the major field of the M.Sc. degree.

AQ:3 = Please specify the major field of the B.Sc. degree.

AQ:4 = Please specify the which degree and major field.

AQ:5 = Please specify the major field of the B.S. and Ph.D. degrees.

AQ:6 = Please specify the year of completion of the Ph.D. degree.

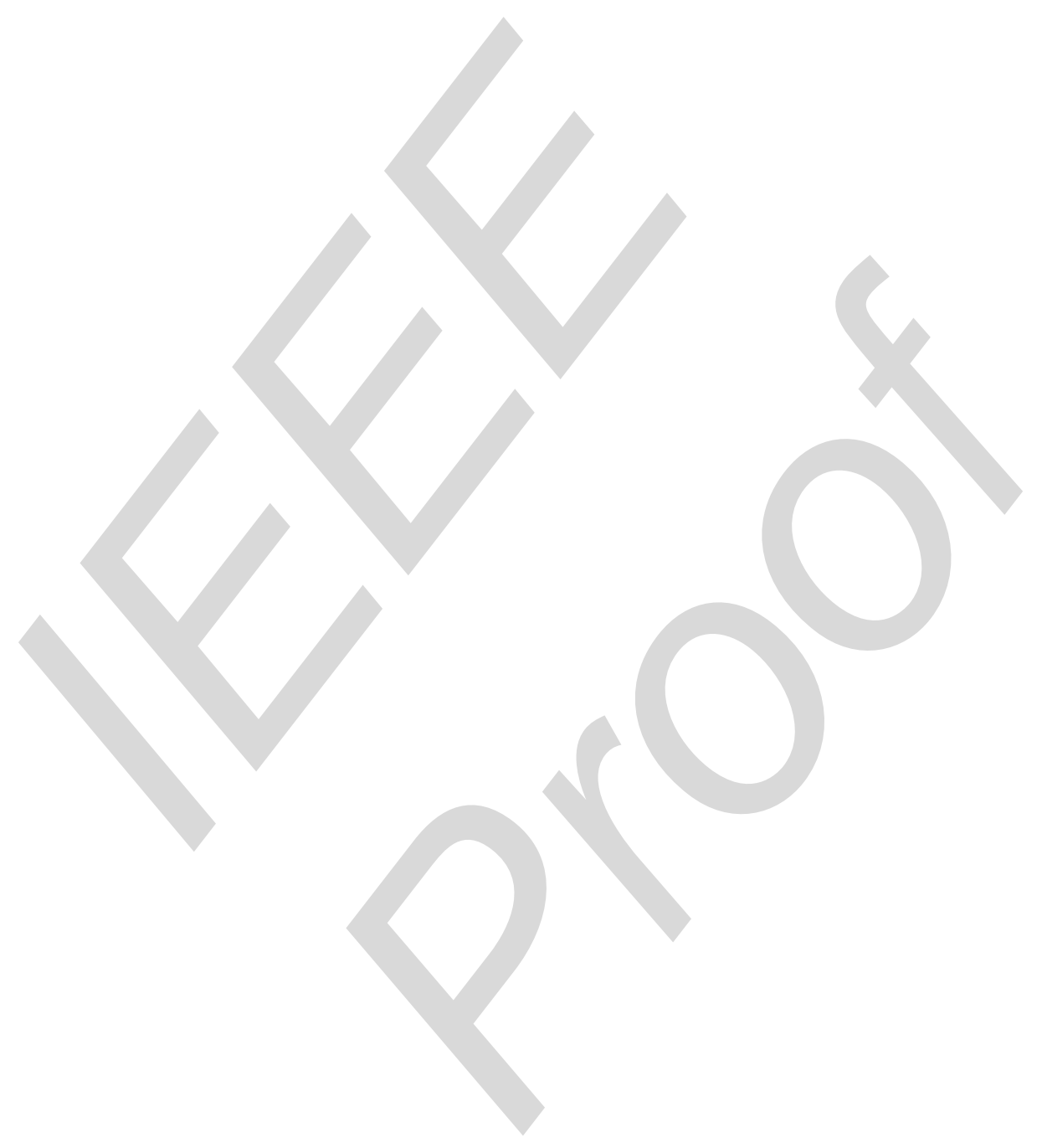

